\title{
Distribution of entanglement and correlations in all finite dimensions
}

\author{
Christopher Eltschka ${ }^{1}$ and Jens Siewert ${ }^{2,3}$ \\ ${ }^{1}$ Institut für Theoretische Physik, Universität Regensburg, D-93040 Regensburg, Germany \\ ${ }^{2}$ Departamento de Química Física, Universidad del País Vasco UPV/EHU, E-48080 Bilbao, Spain \\ ${ }^{3}$ IKERBASQUE Basque Foundation for Science, E-48013 Bilbao, Spain \\ May 16, 2018
}

The physics of a many-particle system is determined by the correlations in its quantum state. Therefore, analyzing these correlations is the foremost task of manybody physics. Any 'a priori' constraint for the properties of the global vs. the local states - the so-called marginals - would help in order to narrow down the wealth of possible solutions for a given many-body problem, however, little is known about such constraints. We derive an equality for correlation-related quantities of any multipartite quantum system composed of finite-dimensional local parties. This relation defines a necessary condition for the compatibility of the marginal properties with those of the joint state. While the equality holds both for pure and mixed states, the pure-state version containing only entanglement measures represents a fully general monogamy relation for entanglement. These findings have interesting implications in terms of conservation laws for correlations, and also with respect to topology.

Correlations between different parts of manyparticle quantum systems are manifestly different from their classical counterparts. The hallmark of the latter is that, if the global state is completely known, so are the local states, that is, the individual states of each particle. For quantum states this may be different: Even if the global state is known with certainty, there may be no information whatsoever regarding the local states such as, for example, in maximally entangled states of two $d$-dimensional systems $[1,2]$.

Often, different degrees of departure from the classical behavior are distinguished, namely entanglement, steerability, and nonlocality [3]. While all these correlation types are based on en- tanglement, nonlocal correlations are regarded as the strongest. This is because all nonlocal states are entangled, but only those for which there are no local models may violate a Bell inequality $[4,5]$. Already such basic considerations reveal that there must exist mathematical tools to characterize quantitative aspects of correlations in a multipartite quantum state. Entanglement measures $[6,7]$ constitute one example of such tools, but there are many others $[8,9]$ and to date it is not clear how to exhaustively characterize quantum correlations, not even for finite-dimensional systems.

Such a characterization can be achieved via mathematical constraints for different correlation functions and/or quantifiers. A Bell inequality is precisely one such constraint: It relates the existence of a local model to the maximum absolute value of a certain linear combination of correlation functions. Another example are so-called monogamy inequalities for entanglement $[10,11,12,13,14]$ or nonlocality $[16,17]$ which establish constraints between the correlations that may coexist in different subsystems of a multipartite quantum system.

It is remarkable that there exist exact relations, that is, equalities between certain entanglement measures in qubit systems. Coffman et al. [10] discovered the now famous relation for pure states $\psi_{3}$ of three qubits (denoted by $A, B$, and $C$ )

$$
\tau_{3}\left(\psi_{3}\right)=\tau_{A}-C_{A B}^{2}-C_{A C}^{2},
$$

where $\tau_{A}$ denotes the linear entropy of the reduced state $\rho_{A}=\operatorname{Tr}_{B C}\left|\psi_{3}\right\rangle\left\langle\psi_{3}\right|, C_{A B}$ is the concurrence of the two-qubit reduced state $\rho_{A B}=$ $\operatorname{Tr}_{C}\left|\psi_{3}\right\rangle\left\langle\psi_{3}\right|$ (analogously for $C_{A C}$ ), and $\tau_{3}\left(\psi_{3}\right)$ is the absolute value of a polynomial local SL (LSL) invariant that depends on the state coefficients of $\psi_{3}$. It turned out only recently [18] that similar relations exists for any $N$-qubit system, the 
simplest being

$$
2\left|H\left(\psi_{N}\right)\right|^{2}=\sum_{k=1}^{N}(-1)^{k+1} \sum_{j_{1}<\ldots<j_{k}} \tau_{A_{j_{1}} \ldots A_{j_{k}}},
$$

where now $\tau_{A_{j_{1}} \ldots A_{j_{k}}}$ is the linear entropy of the reduced state of qubits $A_{j_{1}}, \ldots, A_{j_{k}}$ and $H\left(\psi_{N}\right)=$ $\left\langle\psi_{N}^{*}\left|\sigma_{2}^{\otimes N}\right| \psi_{N}\right\rangle$ is another polynomial LSL invariant [20,7] (here $\left|\psi_{N}^{*}\right\rangle$ is the complex conjugate of $\left|\psi_{N}\right\rangle$ and $\sigma_{2}$ is the second Pauli matrix). Strikingly, all of the quantities in Eqs. (1), (2) are measures for entanglement, either of the individual party (the linear entropies and the concurrences) or in the global state (the polynomial LSL invariants). These relations impose rigid constraints between the properties of the global and the reduced states, and are therefore intimately connected with the quantum marginal problem [19]. However, since their validity appears to be restricted to qubit systems, it is not clear whether they bear any relevance to a general physical setting.

This is the motivation for the present work: We show that the analogue of Eq. (2) holds for any multipartite system of finite-dimensional constituents. In passing, by this relation a local unitary invariant of the pure state is introduced, which we term distributed concurrence, because it describes the distribution of bipartite entanglement in the pure state and is a natural generalization both of the bipartite concurrence and of $\left|H\left(\psi_{N}\right)\right|$ in Eq. (2).

\section{Universal state inversion}

\subsection{Definition}

The key ingredient in our investigation is the operator obtained by applying the so-called universal state inversion [21, 22], see also Refs. [23, 24, 25, 26]. Consider a $d$-dimensional Hilbert space $\mathcal{H}$ and $\mathcal{B}(\mathcal{H})$, the set of bounded positive semidefinite operators on $\mathcal{H}$. Horodecki et al. [21] defined the operator

$$
S_{d}(\mathcal{O})=\operatorname{Tr}(\mathcal{O}) \mathbb{1}-\mathcal{O}, \quad \mathcal{O} \in \mathcal{B}(\mathcal{H})
$$

which was used by Rungta et al. [22] to write the concurrence of a state $\psi \in \mathcal{H}_{A} \otimes \mathcal{H}_{B}$ as

$$
\begin{aligned}
C(\psi) & =\sqrt{\left\langle\psi\left|S_{d_{A}} \otimes S_{d_{B}}(|\psi\rangle\langle\psi|)\right| \psi\right\rangle} \\
& =\sqrt{2-\operatorname{Tr}_{A} \rho_{A}^{2}-\operatorname{Tr}_{B} \rho_{B}^{2}},
\end{aligned}
$$

(where $d_{j}=\operatorname{dim} \mathcal{H}_{j}, j=A, B ; \rho_{A}=\operatorname{Tr}_{B}|\psi\rangle\langle\psi|$, $\rho_{B}=\operatorname{Tr}_{A}|\psi\rangle\langle\psi|$ are the reduced states for the parties). They termed the operator $S_{d}$ the $u n i$ versal state inverter. We will show below that, for non-Hermitian operators $\mathcal{O}$, the appropriate definition of the single-system state inverter is $\tilde{\mathcal{O}}=\operatorname{Tr}\left(\mathcal{O}^{\dagger}\right) \mathbb{1}-\mathcal{O}^{\dagger}$ (see Section 1.4).

It is straightforward to generalize universal state inversion toward density matrices $\rho$ of $N$ partite systems, i.e., $\rho \in \mathcal{B}\left(\mathcal{H}_{1} \otimes \ldots \otimes \mathcal{H}_{N}\right)$, $X \in\{1, \ldots, N\}$

$$
\begin{aligned}
\tilde{\rho} & =\mathcal{S}_{d_{1}} \mathcal{S}_{d_{2}} \ldots \mathcal{S}_{d_{n}}(\rho) \\
& =\left[\prod_{X=1}^{N}\left(\operatorname{Tr}_{X}(\cdot) \otimes \mathbb{1}_{X}-\mathbb{1}\right)\right] \rho,
\end{aligned}
$$

where $\mathbb{1}_{X}$ denotes the identity on a single subsystem $X$, as opposed to $\mathbb{1}$, the identity on the full system. We use the tilde notation for the inverted state $\tilde{\rho}$ in order to make reference to Wootters' notation in Ref. [27], Eq. (5), because that definition for two qubits is naturally generalized by Eq. (7), as we will see below. Note that if the dimension of any party is larger than 2 , the trace of $\tilde{\rho}$ is larger than 1 . Nonetheless we will call $\tilde{\rho}$ the 'tilde state' if no ambiguity is possible.

The operator $\tilde{\rho}$ appears quite frequently in algebraic considerations regarding properties of quantum states, in particular, entanglement. Yet it is not obvious to attribute to it a physical significance beyond the interpretation of Eq. (3) given in Ref. [22]: It takes a pure state to the maximally mixed state in the subspace orthogonal to the pure state, multiplied by the dimension of that subspace. The latter statement can be reexpressed in the following way: Geometrically, the single-system inverter maps a pure state of a $d$-dimensional system to the barycenter of the hypersurface opposite to it, that is, the convex hull of those pure states which, together with the original state, form an orthonormal basis. The result is then scaled with a prefactor $(d-1)$, cf. Fig. 1 .

This geometrical interpretation can be extended to the multipartite case. To this end, consider a state of the computational basis $\left|j_{1} \ldots j_{N}\right\rangle \in \mathcal{H}_{1} \otimes \cdots \otimes \mathcal{H}_{N}$. Then we have

$$
\left|j_{1} \ldots \widehat{\left.j_{N}\right\rangle\left\langle j_{1}\right.} \ldots j_{N}\right|=\bigotimes_{k=1}^{N}\left(\mathbb{1}_{k}-\left|j_{k}\right\rangle\left\langle j_{k}\right|\right),
$$

which corresponds to the barycenter of the hypersurface formed by all those states of the multipartite computational basis whose $k$-th tensor 
factor is different from $\left|j_{k}\right\rangle$. This state is multiplied by a factor $\prod_{k=1}^{N}\left(d_{k}-1\right)$ that keeps track of the total dimension of that hypersurface.

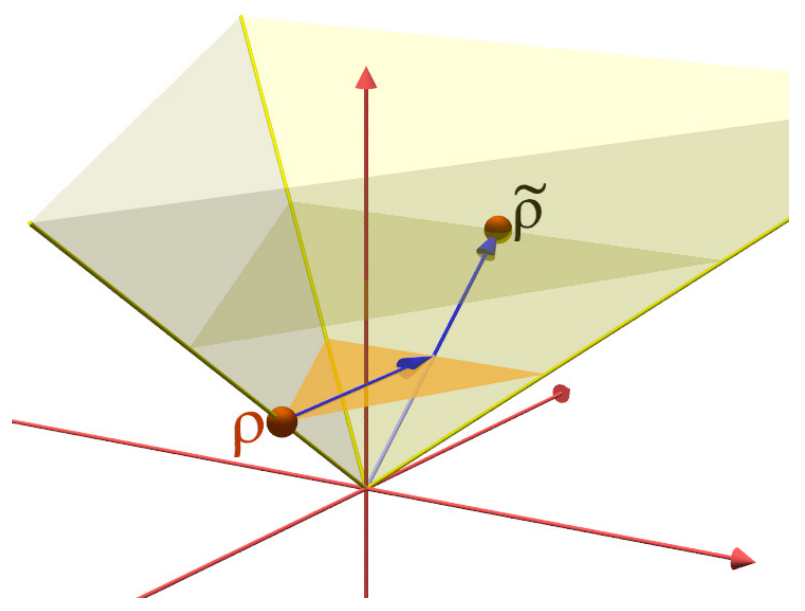

Figure 1: Illustration of the action of the state inverter on a pure state $\rho$ in dimension $d=3$. The orange triangle is a section through the qutrit Bloch body containing the pure state $\rho$ at one corner (orange sphere). The other two corners of the triangle are pure states of which $\tilde{\rho}$ is a linear combination. The vertical axis denotes the multiples of the identity matrix determining the trace. All density matrices have trace 1 , thus the Bloch body is located at a constant height. The axis crosses the Bloch body in the completely mixed state. The state inverter maps the pure state to the state lying furthest on the opposite site of the completely mixed state, as shown by the blue arrow crossing the vertical axis. This state is an equal mixture of the other two pure states in the triangle. At the same time, it scales the vector by the factor $(d-1)=2$, as indicated by the second blue arrow. This scaling maps the Bloch body to a copy at height $(d-1)$, shown as semitransparent yellow triangle. The resulting operator $\tilde{\rho}$ is again marked by a small orange sphere.

\subsection{Representation in terms of reduced states}

There are several noteworthy ways to explicitly write the state $\tilde{\rho}$. The first and obvious possibility is to expand the operator product in Eq. (7) which leads to an alternating sum over all bipartitions $A$ [23]

$$
\tilde{\rho}=\sum_{A}(-1)^{|A|}\left(\operatorname{Tr}_{\bar{A}} \rho\right) \otimes \mathbb{1}_{\bar{A}} .
$$

Here $A$ denotes a subset of $\{1, \ldots, N\},|A|$ is the number of elements in $A, \bar{A}$ is the complement of the subset $A$, and $\rho_{A}=\operatorname{Tr}_{\bar{A}} \rho$ is the reduced density matrix of the parties in the subset $A$.

\subsection{Relation to the Bloch representation of the original state}

A second important way to write $\tilde{\rho}$ is its Bloch representation. In order to obtain it we first consider the Bloch representation of $\rho$ itself. An $N$ qudit state $\rho$ can be written as

$$
\begin{aligned}
\rho & =\frac{1}{d_{1} \cdots d_{N}} \sum_{j_{1} \ldots j_{N}} r_{j_{1} \ldots j_{N}} \mathrm{~g}_{j_{1}} \otimes \cdots \otimes \mathrm{g}_{j_{N}} \\
& =\frac{1}{d_{\text {tot }}}\left(\mathbb{1}+P_{1}+P_{2}+\ldots+P_{N}\right) .
\end{aligned}
$$

Here, $\left\{\mathrm{g}_{j_{k}}\right\}$ are sets of $d_{k}^{2}-1$ traceless generators of $\mathrm{SU}\left(d_{k}\right), \mathrm{g}_{j_{k}}=\mathbb{1}_{k}$ for $j_{k}=0, r_{j_{1} \ldots j_{N}} \in \mathbb{C}$. Moreover, $d_{\text {tot }}=d_{1} \cdots d_{N}$ is the total dimension of the Hilbert space. We use the symbol $P_{q}$ for the sum of all those terms in Eq. (10) that contain the same number $q$ of notrivial generators.

The Bloch representation of $\tilde{\rho}$ is particularly nice if all subsystem dimensions are equal, i.e., $d_{1}=d_{2}=\ldots=d_{N} \equiv d$ and $d_{\text {tot }}=d^{N}$. In order to avoid cumbersome notation we restrict ourselves to this case. Then it is easy to see by directly applying the product form of the state inversion operator Eq. (7) to each term in the Bloch representation Eq. (11) separately that

$$
\begin{aligned}
\tilde{\rho}=\frac{1}{d^{N}} & {\left[(d-1)^{N} \mathbb{1}-(d-1)^{N-1} P_{1}+\right.} \\
& +(d-1)^{N-2} P_{2}-+\ldots+ \\
& \left.+(-1)^{N-1}(d-1) P_{N-1}+(-1)^{N} P_{N}\right] .
\end{aligned}
$$

It is obvious from Eq. (12) that for $d>2$ the universal state inverter is not a trace-preserving map. Further, it shows that only for $d=2$ the tilde state of a pure state $\Pi_{N \text { qubit }}$ is pure as well, because in that case we have for the purity of the tilde state

$$
\begin{aligned}
\left(\operatorname{Tr} \tilde{\Pi}_{N \text { qubit }}\right)^{2} & =\operatorname{Tr} \tilde{\Pi}_{N \text { qubit }}^{2} \\
& =\frac{1}{2^{N}} \sum_{j_{1} \ldots j_{N}}\left|r_{j_{1} \ldots j_{N}}\right|^{2} \\
& =1 .
\end{aligned}
$$

\subsection{Explicit generator representation}

Finally, we want to find a representation of $\tilde{\rho}$ in terms of generators of the unitary group. To 
this end, we use the generalized Gell-Mann matrices [28] in $d$ dimensions

$$
\begin{aligned}
\mathrm{x}_{k l}^{(d)} & =\sqrt{\frac{d}{2}}(|k\rangle\langle l|+| l\rangle\langle k|) \\
\mathrm{y}_{k l}^{(d)} & =\sqrt{\frac{d}{2}}(-\mathrm{i}|k\rangle\langle l|+\mathrm{i}| l\rangle\langle k|) \\
\mathrm{z}_{l}^{(d)} & =\sqrt{\frac{d}{l(l+1)}}\left(-l|l\rangle\left\langle l\left|+\sum_{j=0}^{l-1}\right| j\right\rangle\langle j|\right)
\end{aligned}
$$

where $0 \leqq k<l<d$. The normalization is chosen such that

$$
\begin{aligned}
\operatorname{Tr}\left(\mathrm{x}_{k l} \mathrm{x}_{m n}\right) & =\operatorname{Tr}\left(\mathrm{y}_{k l} \mathrm{y}_{m n}\right)=d \delta_{k m} \delta_{l n}, \\
\operatorname{Tr}\left(\mathrm{z}_{l} \mathrm{z}_{n}\right) & =d \delta_{l n} .
\end{aligned}
$$

Note that, up to a factor, these are the usual $d$ dimensional Gell-Mann matrices.

Just as above in Eq. (10) it is useful to include the identity matrix, as this gives a complete basis for the Hermitian matrices. Sometimes we will use a uniform labeling with only one index and therefore introduce also the alternative form

$$
\begin{aligned}
\mathrm{h}_{0}^{(d)} & =\mathbb{1} \\
\mathrm{h}_{l^{2}+2 k}^{(d)} & =\mathrm{x}_{k l} \\
\mathrm{~h}_{l^{2}+2 k+1}^{(d)} & =\mathrm{y}_{k l} \\
\mathrm{~h}_{l^{2}+2 l}^{(d)} & =\mathrm{z}_{l}
\end{aligned}
$$

where the index corresponds to the usual numbering of the Gell-Mann matrices. Relations (14) then simplify to

$$
\operatorname{Tr}\left(\mathrm{h}_{k} \mathrm{~h}_{l}\right)=d \delta_{k l} .
$$

For the following considerations we need two formulae for the single-system density matrix $\rho \in$ $\mathcal{B}(\mathcal{H})$ with $\operatorname{dim} \mathcal{H}=d$ (a proof is given in Appendix A)

$$
\begin{aligned}
(\operatorname{Tr} \rho) \mathbb{1} & =\frac{1}{d} \sum_{k=0}^{d^{2}-1} \mathrm{~h}_{k} \rho \mathrm{h}_{k} \\
\rho^{T} & =\frac{1}{d} \sum_{k=0}^{d^{2}-1} \mathrm{~h}_{k}^{T} \rho \mathrm{h}_{k} .
\end{aligned}
$$

By applying these relations to Eq. (3) we find for the tilde state of a single system

$$
\begin{aligned}
\tilde{\rho} & =(\operatorname{Tr} \rho) \mathbb{1}-\rho \\
& =\left[(\operatorname{Tr} \rho) \mathbb{1}-\rho^{T}\right]^{*} \\
& =\frac{1}{d} \sum_{k=0}^{d^{2}-1}\left[\mathrm{~h}_{k} \rho \mathrm{h}_{k}-\mathrm{h}_{k}^{T} \rho \mathrm{h}_{k}\right]^{*} .
\end{aligned}
$$

To proceed from here, we note that the generators $\mathrm{y}_{k l}$ change their sign on transposition, while all other generators remain unchanged under that operation. Therefore the sum simplifies to

$$
\begin{aligned}
\tilde{\rho} & =\frac{2}{d} \sum_{k=0}^{d-2} \sum_{l=k+1}^{d-1}\left(\mathrm{y}_{k l} \rho \mathrm{y}_{k l}\right)^{*} \\
& =\frac{2}{d} \sum_{k=0}^{d-2} \sum_{l=k+1}^{d-1} \mathrm{y}_{k l} \rho^{*} \mathrm{y}_{k l} .
\end{aligned}
$$

It is straightforward to extend the result (20) to states of multipartite systems $\rho \in \mathcal{B}\left(\mathcal{H}_{1} \otimes \cdots \otimes\right.$ $\left.\mathcal{H}_{N}\right)$. For this purpose, we introduce the simplified notation

$$
\begin{aligned}
k & =\left(k_{1}, \ldots, k_{N}\right) \\
\mathrm{y}_{k l} & =\mathrm{y}_{k_{1} l_{1}} \otimes \mathrm{y}_{k_{2} l_{2}} \otimes \cdots \otimes \mathrm{y}_{k_{N} l_{N}} \\
\sum_{k<l}^{d-1} & \equiv \sum_{k_{1}=0}^{d_{1}-2} \sum_{l_{1}=k_{1}+1}^{d_{1}-1} \cdots \sum_{k_{N}=0}^{d_{N}-2} \sum_{l_{N}=k_{N}+1}^{d_{N}-1}
\end{aligned}
$$

and can then write

$$
\tilde{\rho}=\frac{2^{N}}{d_{\text {tot }}} \sum_{k<l}^{d-1} \mathrm{y}_{k l} \rho^{*} \mathrm{y}_{k l} .
$$

This is a remarkable result, not only because of its simplicity. It makes explicit that universal state inversion is an antilinear operation. In fact, $\tilde{\rho}$ in Eq. (21) is in all respects the natural generalization of Wootters' tilde state. We note also that for pure states $\rho_{\psi}=|\psi\rangle\langle\psi| \equiv \Pi_{\psi}$ the tilde state $\tilde{\Pi}_{\psi}$ in general is mixed, only for $d_{k}=2$ the generator sum contains a single term, and $\tilde{\Pi}_{\psi}$ becomes pure as well.

Finally, it is immediately clear from Eq. (21) that the tilde state $\tilde{\rho}$ is positive: With $\rho$, also $\rho^{*}$ is positive and therefore $\rho^{*}=\sqrt{\rho^{*}} \cdot \sqrt{\rho^{*}}$, so that

$$
\begin{aligned}
\tilde{\rho} & =\frac{2^{N}}{d_{\mathrm{tot}}} \sum_{k<l}^{d-1} \mathrm{y}_{k l} \sqrt{\rho^{*}} \sqrt{\rho^{*}} \mathrm{y}_{k l} \\
& =\frac{2^{N}}{d_{\mathrm{tot}}} \sum_{k<l}^{d-1}\left(\sqrt{\rho^{*}} \mathrm{y}_{k l}\right)^{\dagger} \sqrt{\rho^{*}} \mathrm{y}_{k l} .
\end{aligned}
$$

That is, $\tilde{\rho}$ is a sum of positive operators and therefore also positive. An important consequence of Eq. (22) is

$$
\operatorname{Tr}(\rho \tilde{\rho})=\operatorname{Tr}\left[(\sqrt{\tilde{\rho}} \sqrt{\rho})^{\dagger}(\sqrt{\tilde{\rho}} \sqrt{\rho})\right] \geqq 0 .
$$




\section{Distributed concurrence}

\subsection{New concurrence-type invariant}

By using the notation $|\psi\rangle\langle\psi| \equiv \Pi_{\psi}$ we can express the concurrence Eq. (5) for the pure state of a bipartite system as $C(\psi)=\sqrt{\operatorname{Tr}\left(\Pi_{\psi} \tilde{\Pi}_{\psi}\right)}$. It is straightforward to extend this definition to pure states of multipartite systems, $\psi \in \mathcal{H}_{1} \otimes \cdots \mathcal{H}_{N}$. We try

$$
\begin{aligned}
C_{D}(\psi)^{2} & =\operatorname{Tr}\left(\Pi_{\psi} \tilde{\Pi}_{\psi}\right) \\
& =\frac{2^{N}}{d_{\text {tot }}} \sum_{k<l}^{d-1} \operatorname{Tr}\left(\Pi_{\psi} \mathrm{y}_{k l} \Pi_{\psi}^{*} \mathrm{y}_{k l}\right) \\
& =(-1)^{N} \frac{2^{N}}{d_{\mathrm{tot}}} \sum_{k<l}^{d-1}\left|\left\langle\psi^{*}\left|\mathrm{y}_{k l}\right| \psi\right\rangle\right|^{2} .
\end{aligned}
$$

Here we see that for odd $N$, the quantity $C_{D}(\psi)$ vanishes identically, for any local dimension, in analogy with the behavior of the $N$-qubit LSL invariant $H\left(\phi_{N}\right)=\left\langle\phi_{N}^{*}\left|\sigma_{2}^{\otimes N}\right| \phi_{N}\right\rangle$ (where $\left.\phi_{N} \in \mathbb{C}_{2}^{\otimes N}\right)$. This is because $\left\langle\phi_{N}^{*}\left|\mathrm{y}_{k l}\right| \phi_{N}\right\rangle=$ $\left\langle\phi_{N}\left|\mathrm{y}_{k l}^{*}\right| \phi_{N}^{*}\right\rangle^{*}=\left\langle\phi_{N}^{*}\left|\left(-\mathrm{y}_{k l}\right)\right| \phi_{N}\right\rangle \equiv 0$ for an odd number $N$ of factors in the tensor product $\mathrm{y}_{k l}$.

We can define now the distributed concurrence

$$
\begin{aligned}
C_{D}(\psi) & =\sqrt{\operatorname{Tr}\left(\Pi_{\psi} \tilde{\Pi}_{\psi}\right)} \\
& =\sqrt{\frac{2^{N}}{d_{\mathrm{tot}}} \sum_{k<l}^{d-1}\left|\left\langle\psi^{*}\left|\mathrm{y}_{k l}\right| \psi\right\rangle\right|^{2}} .
\end{aligned}
$$

Since the universal state inverter Eq. (6) commutes with local unitary operations [22], the distributed concurrence is a local unitary invariant. We will show below that $C_{D}(\psi)$ in certain cases is also an entanglement monotone.

Again, the quantity $C_{D}(\psi)$ generalizes the known concurrence-type quantities in the most natural way: For $N$-qubit states $\phi_{N}$ the LSL invariant $\left|H\left(\phi_{N}\right)\right|$ is obtained, in particular for $N=$ 2 we get Wootters' concurrence $\left|\left\langle\phi_{2}^{*}\left|\sigma_{2} \otimes \sigma_{2}\right| \phi_{2}\right\rangle\right|$. On the other hand, for two parties in dimensions $d_{1}=d_{2}>2$ we get the standard $d \times d$ concurrence, Eq. (5) (cf. also Ref. [29, 30, 31]).

Concluding this section, we note that the operator $\Pi_{\psi} \tilde{\Pi}_{\psi} \Pi_{\psi}$ is proportional to $\Pi_{\psi}$ because of the projector property of $\Pi_{\psi}$. Then, by virtue of Eq. (24), we have

$$
\Pi_{\psi} \tilde{\Pi}_{\psi} \Pi_{\psi}=C_{D}(\psi)^{2} \Pi_{\psi}
$$

2.2 Distributed concurrence is an entanglement monotone for local dimensions smaller than four

In order to investigate the monotone property of the distributed concurrence we need to study whether or not, for pure states, $C_{D}(\psi)$ can increase under the action of separable Kraus operators $O_{j}=A_{1}^{(j)} \otimes \ldots \otimes A_{N}^{(j)}\left(\right.$ where $\left.\sum_{j} O_{j}^{\dagger} O_{j}=\mathbb{1}\right)$, which represent stochastic local operations and classical communication (SLOCC) [32, 33]. If $C_{D}$ is an entanglement monotone for pure states, it can be extended to the mixed states, as usual, through the convex roof [34].

As the distributed concurrence acts symmetrically on the parties it suffices to consider actions on the first party only, $\mathcal{A}_{j}=A_{j} \otimes \mathbb{1}_{2} \otimes \ldots \otimes \mathbb{1}_{N}$. We may assume that the number of parties $N$ is even and that the reduced density matrix of the first party has rank $r_{1}>1$ because otherwise the monotone property is trivially valid. Further, since all generalized measurements may be composed from two-outcome measurements, we will consider Kraus operators with $A_{1}^{\dagger} A_{1}+A_{2}^{\dagger} A_{2}=\mathbb{1}$. Then, for $\psi \in \mathcal{H}_{1} \otimes \cdots \otimes \mathcal{H}_{N}$ we need to show

$$
\begin{aligned}
C_{D}(\psi) & \geqq p_{1} C_{D}\left(\frac{\mathcal{A}_{1} \psi}{\sqrt{p_{1}}}\right)+p_{2} C_{D}\left(\frac{\mathcal{A}_{2} \psi}{\sqrt{p_{2}}}\right) \\
& \geqq C_{D}\left(\mathcal{A}_{1} \psi\right)+C_{D}\left(\mathcal{A}_{2} \psi\right)
\end{aligned}
$$

with $p_{j}=\operatorname{Tr}\left(\mathcal{A}_{j} \Pi_{\psi} \mathcal{A}_{j}^{\dagger}\right)$. In the second line we have used the property $C_{D}(\alpha \psi)=|\alpha|^{2} C_{D}(\psi)$.

We now rewrite Eq (27) by using the decomposition $\Pi_{\psi}=\sum_{j k}\left|e_{j} f_{j}\right\rangle\left\langle e_{k} f_{k}\right|$, where $\left\{\left|e_{j}\right\rangle\right\}$ are orthonormal vectors that span the Hilbert space of the first party and are chosen such that they diagonalize the operator $\mathcal{A}_{1}^{\dagger} \mathcal{A}_{1}$. The vectors $\left\{\left|f_{j}\right\rangle\right\}$ span the Hilbert space of the last $(N-1)$ parties in $\psi$ and are not necessarily orthogonal or normalized, but they obey $\sum_{j}\left\langle f_{j} \mid f_{j}\right\rangle=1$. We obtain

$$
\begin{aligned}
\operatorname{tr}\left(\Pi_{\psi} \tilde{\Pi}_{\psi}\right) & =2 \sum_{j k} F_{k j j k} \\
\operatorname{tr}\left(A_{1} \Pi_{\psi} A_{1}^{\dagger}\right)\left(\widetilde{A_{1} \Pi_{\psi} A_{1}^{\dagger}}\right) & =2 \sum_{k l} F_{k j j k} D_{j} D_{k}
\end{aligned}
$$

where we have used the definitions

$$
\begin{aligned}
D_{k} \delta_{j k} & \equiv\left\langle e_{k}\left|\mathcal{A}_{1}^{\dagger} \mathcal{A}_{1}\right| e_{j}\right\rangle \quad, 0 \leqq D_{k} \leqq 1 \\
F_{k l m j} & \equiv\left\langle f_{k}\left|\left(\mid \widehat{\left.f_{l}\right\rangle\left\langle f_{m}\right|}\right)\right| f_{j}\right\rangle .
\end{aligned}
$$


Note that $F_{k l m j}=F_{l k j m}=-F_{l k m j}$, which follows with the explicit inversion formula (21). Finally, $p_{1}=\operatorname{Tr}\left(\mathcal{A}_{1} \Pi_{\psi} \mathcal{A}_{1}^{\dagger}\right)=\sum_{j} D_{j}\left\langle f_{j} \mid f_{j}\right\rangle$ and $p_{2}=$ $\operatorname{Tr}\left(\mathcal{A}_{2} \Pi_{\psi} \mathcal{A}_{2}^{\dagger}\right)=\sum_{j}\left(1-D_{j}\right)\left\langle f_{j} \mid f_{j}\right\rangle$.

With all this, we can transform Eq. (27) into the equivalent inequality

$$
\begin{aligned}
\sqrt{\sum_{j k} F_{j k k j}} & \geqq \sqrt{\sum_{j k} F_{j k k j} D_{j} D_{k}}+ \\
& +\sqrt{\sum_{j k} F_{j k k j}\left(1-D_{j}\right)\left(1-D_{k}\right)} .
\end{aligned}
$$

Now it is straightforward to prove the monotone property for the well-known cases $d=2, N$ arbitrary, and $N=2, d$ arbitrary, as we show in Appendix B. For the general case, we continue transforming Eq. (28) by subtracting the second term on the right, squaring and simplifying the result, to obtain

$$
\left(\sum_{j k} w_{j k} D_{j}\right)^{2} \geqq \sum_{j k} w_{j k} D_{j} D_{k},
$$

with the weights $w_{j k} \equiv F_{j k k j} /\left(\sum_{l m} F_{l m m l}\right)$. Note that, if the denominator here were zero, the monotone property would be trivially satisfied. It is possible to construct examples of $\left\{D_{k}\right\}$ and $\left\{F_{l m m l}\right\}$ such that inequality (29) is violated for $\operatorname{dim} \mathcal{H}_{1}=4$ (see Appendix C). Therefore, $C_{D}$ is not an entanglement monotone if a party has local dimension $d_{j} \geqq 4$ and the party number $N \geqq 4$. Similarly, such sets can be found to show that $C_{D}^{2}$ is not a monotone for $d_{j} \geqq 3, N \geqq 4$.

Remarkably, however, Eq. (29) does hold for $\operatorname{dim} \mathcal{H}_{1}=3$, that is, $C_{D}$ is an entanglement monotone for any number of parties if all local dimensions are not larger than three. This behavior resembles that of LSL invariants for which it is known that the monotone property depends on the homogeneity degree [35] and, in fact, also on the local dimension.

For the proof of Eq. (29) in the case $\operatorname{dim} \mathcal{H}_{1}=3$ we collect only the $D_{j}^{2}$ terms on the left and multiply both sides by 4 . Moreover, we use $2\left(w_{01}+w_{02}+w_{12}\right)=1$ and obtain

$$
\begin{aligned}
\left(1-2 w_{12}\right)^{2} D_{0}^{2}+\left(1-2 w_{02}\right)^{2} D_{1}^{2}+\left(1-2 w_{01}\right)^{2} D_{2}^{2} \geqq & D_{0} \sqrt{2\left(2 w_{01}\right)-\left(1-2 w_{12}\right)\left(1-2 w_{02}\right)^{2}} D_{1}+ \\
& \left.+D_{0} \sqrt{2\left(2 w_{02}\right)-\left(1-2 w_{12}\right)\left(1-2 w_{01}\right.}\right)^{2} D_{2}+ \\
& +D_{1} \sqrt{2\left(2 w_{12}\right)-\left(1-2 w_{01}\right)\left(1-2 w_{02}\right)} D^{2}+ \\
& +D_{1}{\sqrt{2\left(2 w_{01}\right)-\left(1-2 w_{12}\right)\left(1-2 w_{02}\right)}}^{2} D_{0}+ \\
& +D_{2}{\left.\sqrt{2\left(2 w_{12}\right)-\left(1-2 w_{02}\right)\left(1-2 w_{01}\right.}\right)^{2} D_{1}+}^{2}{ }^{2} D_{2}{\sqrt{2\left(2 w_{02}\right)-\left(1-2 w_{12}\right)\left(1-2 w_{01}\right)} D_{0} .}
\end{aligned}
$$

The peculiar way of writing Eq. (30) indicates how one can see that this is precisely a CauchySchwarz inequality, and therefore is valid. This concludes the proof that Eq. (29) holds for $\operatorname{dim} \mathcal{H}_{1}=3$.

To summarize this section (see also Fig. 2), we have found that both $C_{D}$ and $C_{D}^{2}$ are entanglement monotones only in the well-known cases and, trivially, for odd number of parties $N$. For local dimensions $d_{j} \leqq 3$ and even party number $N, C_{D}$ is an entanglement monotone, but not $C_{D}^{2}$.
For local dimensions $d_{j} \geqq 4$ neither $C_{D}$ nor $C_{D}^{2}$ is an entanglement monotone for any even $N>2$.

\section{Distribution of correlations and monogamy of entanglement}

In Ref. [18], the monogamy relation Eq. (2) for $N$ qubits was derived. The strategy there was to write down a local SL invariant for an $N$-qubit state $\rho_{N}$ via combining $\rho_{N}$ and $\tilde{\rho}_{N}$, and, by ex- 


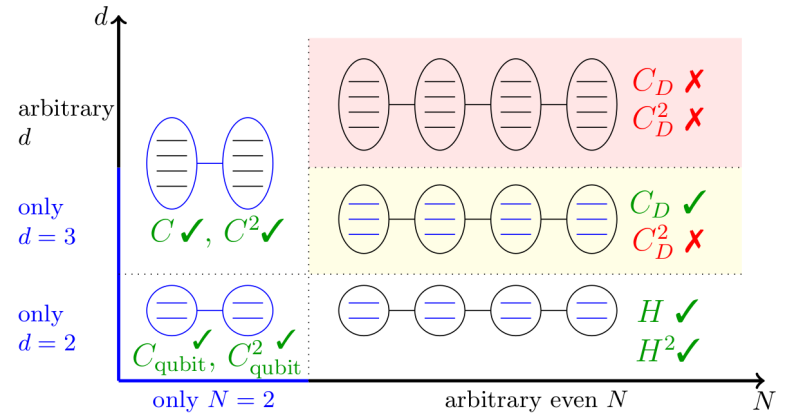

Figure 2: The local unitary invariant $C_{D}$, cf. Eq. (24), termed distributed concurrence, naturally generalizes the well-known cases of bipartite concurrence $d \geqq 2, N=2$ $[22,29]$ and $H$ invariant for $N$-qubit system [20]. While in those cases both $C_{D}$ and $C_{D}^{2}$ are monotones, the general case $d>2, N>2$ is different: $C_{D}$ (but not $C_{D}^{2}$ ) is an entanglement monotone only if all local dimensions $d \leqq 3$. If there is one subsystem of dimension at least four, neither $C_{D}$ nor $C_{D}^{2}$ are entanglement monotones.

panding that expression in terms of Bloch components to obtain an identity which connects the invariant (for the global state) with combinations of local quantities (linear entropies). Now we ask whether one can obtain a new result by generalizing Eq. (2) in the frame of the present formalism. The answer is affirmative, as we will show.

We consider a state $\rho$ of a finite-dimensional multipartite quantum system $\rho \in \mathcal{B}\left(\mathcal{H}_{1} \otimes \ldots \otimes\right.$ $\left.\mathcal{H}_{N}\right)$. Clearly, $\operatorname{Tr}(\rho \tilde{\rho})$ is a local unitary invariant. We expand this expression as a sum over all bipartite splits $A \mid \bar{A}$ by applying Eq. (9)

$$
\begin{aligned}
\operatorname{Tr}(\rho \tilde{\rho}) & =1+\sum_{|A|>0}(-1)^{|A|} \operatorname{Tr}\left[\rho \operatorname{Tr}_{\bar{A}} \rho \otimes \mathbb{1}_{\bar{A}}\right] \\
& =1+\sum_{|A|>0}(-1)^{|A|} \operatorname{Tr}_{A}\left[\operatorname{Tr}_{\bar{A}} \rho\right]^{2} \\
& =\frac{1}{2} \sum_{|A|>0}(-1)^{|A|+1} 2\left(1-\operatorname{Tr} \rho_{A}^{2}\right)
\end{aligned}
$$

By using the definition of the linear entropy on the partition $A, \tau_{A}=2\left(1-\operatorname{Tr} \rho_{A}^{2}\right)$ we obtain

$$
0 \leqq 2 \operatorname{Tr}(\rho \tilde{\rho})=\sum_{|A|>0}(-1)^{|A|+1} \tau_{A} .
$$

This is an equality for the distribution of correlations and holds both for pure and for mixed states of any finite-dimensional multipartite system. The general validity of Eq. (31) is quite remarkable: In Ref. [18] it was derived by using a qubit-specific formalism, therefore its full generality could not be expected.
It is in order here to recall the quantum marginal problem [19], which poses the question whether or not a given set of marginal states is compatible with a (possibly unique) global state. The equality (31) represents a simple necessary (but not sufficient) condition for a favorable answer to that question.

It is interesting here to consider the special case of mixed states $\rho_{A B C}$ of three-parties A, B, and C. From Eq. $(31)$ and $\operatorname{Tr}(\rho \tilde{\rho}) \geqq 0$ we find

$$
\tau_{A}+\tau_{B}+\tau_{C}+\tau_{A B C} \geqq \tau_{A B}+\tau_{A C}+\tau_{B C} .
$$

This reminds of the strong subadditivity inequality for the von-Neumann entropy $S$ [37], which reads $S_{A B C}+S_{B} \leqq S_{A B}+S_{B C}$. However, inequality (32) is symmetrized between the parties and has the opposite direction.

We emphasize that the $\tau_{A}$ in Eq. (31) are simply the linear entropies of the reduced states $\rho_{A}$, so they are not entanglement measures. However, if we consider pure states $|\psi\rangle\langle\psi| \equiv \Pi_{\psi}$, we note $\tau_{A}(\psi)=0$ for $|A|=N$, and the equality reads

$$
\begin{aligned}
0 \leqq 2 C_{D}^{2}(\psi) & =\sum_{|A|>0}(-1)^{|A|+1} \tau_{A}(\psi) \\
& =\sum_{N>|A|>0}(-1)^{|A|+1} C_{A \mid \bar{A}}^{2}(\psi) .
\end{aligned}
$$

Here we denote by $C_{A \mid \bar{A}}(\psi)$ the bipartite concurrence on the split $A \mid \bar{A}$ according to Eq. (5). We see that Eq. (33) represents a true monogamy relation, because all the terms on the right-hand side are entanglement measures. If the local dimensions are not larger than three, also $C_{D}$ is an entanglement measure, so that we even have a monogamy equality. Thus, Eq. (33) is a constraint for the distribution of entanglement across all bipartitions of an arbitrary multipartite pure state of finite dimensionality. For odd $N$ the equality is trivial, because the purities on complementary partitions $A$ and $\bar{A}$ enter it with a different sign, confirming that $C_{D}(\psi)=0$ in this case. We note that aspects of the results discussed here and summarized in Eqs. (31), (33) were obtained in Ref. [36].

It is an interesting feature of Eqs. (33), (32) that they describe all local parties in a symmetric manner, whereas the Coffman-Kundu-Wootters identity Eq. (1) or the strong subadditivity inequality single out one party. Our way of deriving the general monogamy relation (33) seems to 
suggest that the symmetric form has a peculiar role, just because the tilde state $\tilde{\rho}$ is symmetric in the local parties as well.

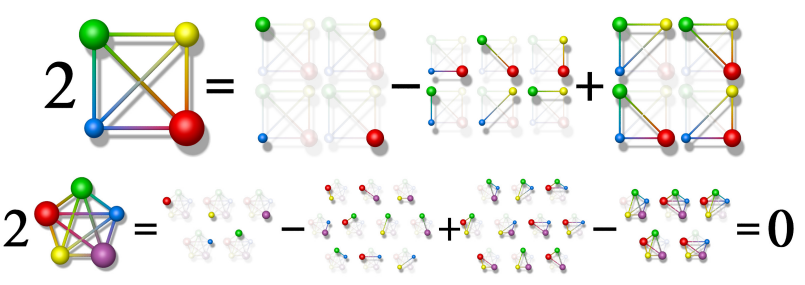

Figure 3: Illustration of the $N$-qudit monogamy relation Eq. (33). The left-hand side symbolizes $C_{D}^{2}$ while, on the right-hand side, there are the representations of the linear entropies of all possible bipartite splits in the set of $N$ qudits.

Top panel: Four qudits. Complementary partitions $A$ and $\bar{A}$ enter with the same sign.

Bottom panel: Five qudits. For odd $N$, the monogamy relation is trivial because the linear entropy of a partition $A$ equals that of its complementary partition $\bar{A}$, but contributes with opposite sign.

\section{Other interesting aspects}

\subsection{Conservation laws for correlations}

In the previous section we have emphasized that in Eq. (31) all terms are local unitary invariants. The left-hand side is locally invariant on each single party, whereas the terms on the right-hand side are locally invariant on their respective bipartition. We explain now that Eq. (31) gives rise to nontrivial unitary invariants on an entire subset of the parties.

To this end, let us consider an example of a four-party state $\rho_{1234}$ where each index denotes the respective party. The reduced state, e.g., of parties $\{13\}$ is $\rho_{13}=\operatorname{Tr}_{24} \rho_{1234}$. Correspondingly, the linear entropy of parties $\{13\}$ is $\tau_{13}=2(1-$ $\left.\operatorname{Tr}_{13} \rho_{13}^{2}\right)$. Then we have

$$
\begin{aligned}
\operatorname{Tr} \rho_{1234} \tilde{\rho}_{1234}= & \tau_{1}+\tau_{2}+\tau_{3}+\tau_{4}-\tau_{12}-\ldots- \\
& -\tau_{34}+\tau_{123}+\tau_{124}+\tau_{134}+\tau_{234}- \\
& -\tau_{1234} .
\end{aligned}
$$

Assume now that the subsystem of parties $\{13\}$ is subject to unitary evolution $U_{13}(t)=\mathbb{1}_{24} \otimes$ $\exp \left(-\mathrm{iH}_{13} t\right)$ generated by a (time-independent) Hamiltonian $\mathrm{H}_{13}$. The full state $\rho_{1234}$ be mixed, e.g., the thermal state at some temperature. Let us collect all the terms in Eq. (34) in which both of the parties 1 and 3 , or none of them, are traced out:

$$
\begin{aligned}
- & \tau_{2}-\tau_{4}+\tau_{13}+\tau_{24}-\tau_{123}-\tau_{134}+\tau_{1234}= \\
= & -\operatorname{Tr} \rho_{1234} \tilde{\rho}_{1234}+\tau_{1}+\tau_{3}-\tau_{12}-\tau_{14}- \\
& -\tau_{23}-\tau_{34}+\tau_{124}+\tau_{234}
\end{aligned}
$$

The left-hand side of Eq. (35) is invariant under the evolution $U_{13}(t)$ while each of the terms on the right will vary in a nontrivial way, as the time evolution generates or degrades correlations of the corresponding parties with the other subsystems. This means Eq. (35) represents a conservation law for correlations. Analogous conservation laws can be found for Hamiltonian subsystem dynamics of any finite-dimensional multipartite state.

\subsection{Relation between distributed concurrence and topology}

A striking property of Eqs. (31) and (33) is their apparent connection with topology. Just the form of those equations hints at the so-called Euler characteristic [38], a number characterizing the structure of a topological space. Yet in the present case the nature of the topological entity, described in particular by Eq. (33) is not really clear. Another problem is that Eq. (33) in general is not a relation between integers, which makes its topological interpretation questionable.

However, there are more indications in the properties of $C_{D}$ that there indeed exists a relation to topology. First, it is known that a closed topological space of odd dimension has Euler characteristic 0. This coincides with the property $C_{D} \equiv 0$ for systems with odd number of parties $N$. Further, the Euler characteristic of a product manifold equals the product of the Euler characteristics of the factors. Again, it is easy to see from the explicit definition of $C_{D}$, Eq. (25), that the distributed concurrence has the same property. Finally, the Euler characteristic bears a close relation with the inclusion-exclusion principle. The derivation of Eq. (33) for qubits in Ref. [18] via the Bloch representation is an explicit application of this principle.

We can only speculate what the topological nature of Eq. (33) is. A tempting interpretation is the following. We regard the $N$ parties as vertices of a standard $(N-1)$-simplex. In a simplex, each 
vertex is connected to each other vertex. This appears to correspond to the distribution of entanglement in a genuinely entangled [33] pure state: A given party is entangled with any other party in such a pure state. In a strict sense there is no distinction, such as 'only pairwise entanglement', as is possible for mixed states. The boundary of an $(N-1)$-simplex is topologically equivalent to a sphere $S^{N-2}$ and therefore has Euler characteristic $1+(-1)^{N-2}$ which corresponds to the behavior of $C_{D}$ (vanishing $C_{D}$ for odd $N$ ). Finally, if the pure state can be written as a product of exactly two factors (with party numbers $k$ and $N-k$ ), the topology would be that of two disjoint simplices, one with $k$ and the other with $N-k$ vertices.

\section{Conclusions}

We have analyzed universal state inversion applied to multipartite quantum states with finitedimensional parties. This map takes pure states to the opposite hypersurface of the state space and therefore algebraically encodes extremal properties of quantum states. We made explicit that this map is antilinear. In the framework of our formalism we were able to derive a universally valid equality, Eq. (31), that relates a local unitary invariant depending on the global state to the linear entropies of all possible reduced states. Remarkably, if we apply this equality to pure states we obtain the first known monogamy relation for bipartite entanglement in a multipartite state, Eq. (33), which is valid for any number of parties and any finite local dimension. These findings appear to have a close relation with the topological properties of multipartite quantum systems, as we have argued in Sec. 4.2. Moreover, our correlation equalities give rise to conservation laws for correlations, which are valid for any type of unitary subsystem dynamics.

The local unitary invariant mentioned above, the distributed concurrence, describes the quantitative distribution of bipartite entanglement in a multipartite pure state. It naturally generalizes the concurrence in the well-known cases, that is, for bipartite systems of arbitrary dimension and for many-qubit systems. Thus, it provides both the mathematical and conceptual link between these hitherto somewhat unrelated quantities, see Eqs (2),(5). Intriguingly, for systems of even party number $N \geqq 4$ the distributed concurrence is an entanglement monotone only below a 'critical' local dimension $d \leqq 3$. Note, however, that for its role as a 'generator' of the monogamy inequality the monotone property of the distributed concurrence is not essential.

Our work shows that, by means of algebraic methods, it is possible to express the mathematical constraints to quantum states, in particular their positivity, in terms of physically relevant quantities and principles. Importantly, our results establish necessary relations between the global and the local states which may be considered as a partial answer in the context of the quantum-marginal problem. Clearly, our result highlights only one specific aspect of correlations, and the question is whether also other mathematical constraints allow for a similar formulation with direct access to their physical meaning.

\section{Acknowledgments}

This work was funded by the German Research Foundation Project EL710/2-1 (C.E.), by Basque Government grant IT986-16, MINECO/FEDER/UE grants FIS2012-36673C03-01 and FIS2015-67161-P and UPV/EHU program UFI $11 / 55$ (J.S.). The authors would like to thank Jaroslav Fabian, Felix Huber, Marcus Huber, Peter D. Jarvis, and Denis Kochan for stimulating discussions, and Klaus Richter for supporting this project. F.H. pointed out to us Refs. [23, 24, 36]. D.K. noticed the apparent similarity between the monogamy relation and the Euler characteristic.

\section{A Proof of useful generator formulae}

The interesting relations (17), (18) are closely linked to the well-known completeness relation for the set of generators $\left\{\mathrm{h}_{k}\right\}$ (see, e.g., [28]). It is instructive to derive all these relations in the same framework, i.e., by consequently using the terminology of quantum information.

An important ingredient is the SWAP operator. Consider two states $|\psi\rangle,|\phi\rangle \in \mathcal{H}$. Then we define

$$
\operatorname{SWAP} \cdot(|\psi\rangle \otimes|\phi\rangle)=|\phi\rangle \otimes|\psi\rangle .
$$

With the computational basis $\{|j\rangle\}$ of $\mathcal{H}$ it is straightforward to show that SWAP = 
$\sum_{j k}|j k\rangle\langle k j|$. Let us denote by $\operatorname{Tr}_{[1]}$ the partial trace over the first tensor factor in $\mathcal{H} \otimes \mathcal{H}$, and correspondingly by $\operatorname{Tr}_{[2]}$ the partial trace over the second factor. Then we find by explicit calculation in the computational basis that for two operators $A, B \in \mathcal{B}(\mathcal{H})$

$$
\begin{aligned}
& \operatorname{Tr}_{[1]}(\text { SWAP } \cdot(A \otimes B))=A \cdot B \\
& \operatorname{Tr}_{[2]}(\text { SWAP } \cdot(A \otimes B))=B \cdot A
\end{aligned}
$$

and

$$
\begin{aligned}
& \operatorname{Tr}_{[1]}((A \otimes B) \cdot \text { SWAP })=B \cdot A \\
& \operatorname{Tr}_{[2]}((A \otimes B) \cdot \text { SWAP })=A \cdot B .
\end{aligned}
$$

Important special cases of these relations are obtained by setting, e.g., $B=\mathbb{1}$,

$$
\operatorname{Tr}_{[1]}(\operatorname{SWAP} \cdot(A \otimes \mathbb{1}))=A .
$$

Now consider a set $\left\{\mathrm{h}_{j}\right\}, j=1 \ldots\left(d^{2}-1\right)$ of traceless Hermitian generators of $\mathrm{SU}(d), \operatorname{Tr}\left(\mathrm{h}_{j} \mathrm{~h}_{k}\right)=$ $d \delta_{j k}, \mathrm{~h}_{0} \equiv \mathbb{1}$, so that we can expand

$$
\begin{aligned}
A & =\frac{1}{d} \sum_{j=0}^{d^{2}-1} a_{j} \mathrm{~h}_{j}=\frac{1}{d} \sum_{j=0}^{d^{2}-1} \operatorname{Tr}\left(\mathrm{h}_{j} A\right) \mathrm{h}_{j} \\
& =\operatorname{Tr}_{[1]}\left[\left(\frac{1}{d} \sum_{j=0}^{d^{2}-1} \mathrm{~h}_{j} \otimes \mathrm{h}_{j}\right) \cdot(A \otimes \mathbb{1})\right] .
\end{aligned}
$$

Because $A$ is an arbitrary operator here, comparison of Eqs. (41), (43) yields the completeness relation for the generators $\mathrm{h}_{j}$

$$
\mathrm{SWAP}=\frac{1}{d} \sum_{j=0}^{d^{2}-1} \mathrm{~h}_{j} \otimes \mathrm{h}_{j} .
$$

With these prelimary considerations we can turn to the proof of the operator relations in Sec. 1.4. Inserting Eq. (44) into the identity

$$
\mathrm{SWAP} \cdot(A \otimes \mathbb{1}) \cdot \mathrm{SWAP}=\mathbb{1} \otimes A
$$

and tracing over the second party gives

$$
\begin{aligned}
\operatorname{Tr}(A) \mathbb{1} & =\frac{1}{d^{2}} \sum_{j, k=0}^{d^{2}-1}\left(\mathrm{~h}_{j} A \mathrm{~h}_{k}\right) \operatorname{Tr}\left(\mathrm{h}_{j} \mathrm{~h}_{k}\right) \\
& =\frac{1}{d} \sum_{j=0}^{d^{2}-1} \mathrm{~h}_{j} A \mathrm{~h}_{j}
\end{aligned}
$$

which proves Eq. (17).

From the explicit representation of SWAP in the computational basis it is obvious that it is related to the maximally entangled state $\left|\Phi^{+}\right\rangle \equiv$ $\frac{1}{\sqrt{d}} \sum_{j}|j j\rangle$ by virtue of partial transposition, e.g., on the first party, $T_{[1]}$,

$$
\begin{aligned}
\operatorname{SWAP}^{T_{[1]}} & =\left(\sum_{j k}|j k\rangle\langle k j|\right)^{T_{[1]}} \\
& =\sum_{j k}|k k\rangle\langle j j| \\
& =d\left|\Phi^{+}\right\rangle\left\langle\Phi^{+}\right|
\end{aligned}
$$

We conclude from Eqs. (44) and (46) that

$$
\operatorname{SWAP}_{[1]}=\frac{1}{d} \sum_{j=0}^{d^{2}-1} \mathrm{~h}_{j}^{T} \otimes \mathrm{h}_{j} .
$$

In analogy with Eqs. (37)-(40) we obtain now (by explicit calculation in the computational basis)

$$
\begin{aligned}
& \operatorname{Tr}_{[1]}\left(d\left|\Phi^{+}\right\rangle\left\langle\Phi^{+}\right| \cdot(A \otimes B)\right)=A^{T} \cdot B \\
& \operatorname{Tr}_{[2]}\left(d\left|\Phi^{+}\right\rangle\left\langle\Phi^{+}\right| \cdot(A \otimes B)\right)=B^{T} \cdot A \\
& \operatorname{Tr}_{[1]}\left((A \otimes B) \cdot d\left|\Phi^{+}\right\rangle\left\langle\Phi^{+}\right|\right)=B \cdot A^{T} \\
& \operatorname{Tr}_{[2]}\left((A \otimes B) \cdot d\left|\Phi^{+}\right\rangle\left\langle\Phi^{+}\right|\right)=A \cdot B^{T} .
\end{aligned}
$$

By using Eq. (48) with $B=\mathbb{1}$, the permutation invariance of $\left|\Phi^{+}\right\rangle$, and Eq. (47) we find

$$
\begin{aligned}
A^{T} & =\operatorname{Tr}_{[1]}\left(d\left|\Phi^{+}\right\rangle\left\langle\Phi^{+}\right| \cdot(A \otimes \mathbb{1})\right) \\
& =\operatorname{Tr}_{[1]}\left(\operatorname{SWAP} \cdot d\left|\Phi^{+}\right\rangle\left\langle\Phi^{+}\right| \cdot(A \otimes \mathbb{1})\right) \\
& =\frac{1}{d} \sum_{j=0}^{d^{2}-1} \operatorname{Tr}_{[1]}\left(\operatorname{SWAP} \cdot\left[\left(\mathrm{h}_{j}^{T} A\right) \otimes \mathrm{h}_{j}\right]\right)
\end{aligned}
$$

and, finally, with Eq. (37)

$$
A^{T}=\frac{1}{d} \sum_{j=0}^{d^{2}-1} \mathrm{~h}_{j}^{T} A \mathrm{~h}_{j},
$$

which proves Eq. (18).

\section{B Proof of monotone property in the known cases}

For the sake of completeness, we present the proof for the monotone property of both $C_{D}$ and $C_{D}^{2}$ in the known cases in the frame of the formalism in Sec. 2.2.

$d=2, \quad N$ arbitrary: Here, there are only two indices $j \in\{0,1\}$ and only one 
value of $F_{0110}=F_{1001}$, so that the inequality (28) becomes $\sqrt{2 F_{0110}} \geqq \sqrt{2 F_{0110} D_{0} D_{1}}+$ $\sqrt{2 F_{0110}\left(1-D_{0}\right)\left(1-D_{1}\right)}$ from which we have

$$
1 \geqq \sqrt{D_{0} D_{1}}+\sqrt{\left(1-D_{0}\right)\left(1-D_{1}\right)}
$$

and further

$$
\left(1-\sqrt{D_{0} D_{1}}\right)^{2} \geqq\left(1-D_{0}\right)\left(1-D_{1}\right)
$$

which is equivalent to

$$
D_{0}+D_{1} \geqq 2 \sqrt{D_{0} D_{1}}
$$

and always fulfilled because of the relation between arithmetic and geometric mean.

The monotone condition Eq. (27) for the square of $C_{D}$ reads

$$
\begin{aligned}
C_{D}^{2}(\psi) & \geqq p_{1} C_{D}^{2}\left(\frac{\mathcal{A}_{1} \psi}{\sqrt{p_{1}}}\right)+p_{2} C_{D}^{2}\left(\frac{\mathcal{A}_{2} \psi}{\sqrt{p_{2}}}\right) \\
& \geqq \frac{C_{D}^{2}\left(\mathcal{A}_{1} \psi\right)}{p_{1}}+\frac{C_{D}^{2}\left(\mathcal{A}_{2} \psi\right)}{p_{2}} .
\end{aligned}
$$

In the proof for $C_{D}^{2}$ we use the probability $p_{1}=$ $\left\langle f_{0} \mid f_{0}\right\rangle D_{0}+\left\langle f_{1} \mid f_{1}\right\rangle D_{1}$ (recall that $\sum_{j}\left\langle f_{j} \mid f_{j}\right\rangle=$ 1 ), and we have to show that

$$
1 \geqq \frac{D_{0} D_{1}}{p_{1}}+\frac{\left(1-D_{0}\right)\left(1-D_{1}\right)}{1-p_{1}} .
$$

This inequality is equivalent to

$$
0 \geqq\left(p_{1}-D_{0}\right)\left(p_{1}-D_{1}\right)
$$

which is always fulfilled as is easily checked with the explicit formula for $p_{1}$.

$N=2, d$ arbitrary: In this case we have

$$
\begin{aligned}
F_{j k k j} & =\left\langle f_{j}\left|\left(\widehat{\left.f_{k}\right\rangle\left\langle f_{k}\right.} \mid\right)\right| f_{j}\right\rangle \\
& =\left\langle f_{j}\left|\left(\operatorname{Tr}\left(\left|f_{k}\right\rangle\left\langle f_{k}\right|\right) \mathbb{1}-\left|f_{k}\right\rangle\left\langle f_{k}\right|\right)\right| f_{j}\right\rangle \\
& =\left\langle f_{j} \mid f_{j}\right\rangle\left\langle f_{k} \mid f_{k}\right\rangle-\left|\left\langle f_{j} \mid f_{k}\right\rangle\right|^{2},
\end{aligned}
$$

because the subnormalized vectors $\left\{\left|f_{j}\right\rangle\right\}$ belong to a single party. and inequality (28) takes the form

$$
\begin{gathered}
\sqrt{1-\sum_{j k}\left|\left\langle f_{j} \mid f_{k}\right\rangle\right|^{2}} \geqq \sqrt{p_{1}^{2}-\sum_{j k}\left|\left\langle f_{j} \mid f_{k}\right\rangle\right|^{2} D_{j} D_{k}}+ \\
+\sqrt{\left(1-p_{1}\right)^{2}-\sum_{j k}\left|\left\langle f_{j} \mid f_{k}\right\rangle\right|^{2}\left(1-D_{j}\right)\left(1-D_{k}\right)}
\end{gathered}
$$

The right-hand side (rhs) of this relation can be upper-bounded by using the concavity of the square root. Subsequent squaring yields

$$
\begin{aligned}
(\mathrm{rhs})^{2} & \leqq 1-\frac{\sum_{j k}\left|\left\langle f_{j} \mid f_{k}\right\rangle\right|^{2} D_{j} D_{k}}{p_{1}}-\frac{\sum_{j k}\left|\left\langle f_{j} \mid f_{k}\right\rangle\right|^{2}\left(1-D_{j}\right)\left(1-D_{k}\right)}{1-p_{1}} \\
& \leqq 1-\sum_{j k}\left|\left\langle f_{j} \mid f_{k}\right\rangle\right|^{2} \frac{p_{1}\left(1-p_{1}\right)+\left(p_{1}-D_{j}\right)\left(p_{1}-D_{k}\right)}{p_{1}\left(1-p_{1}\right)} .
\end{aligned}
$$

Hence, in order to prove the monotone property it remains to show that

$$
0 \leqq \sum_{j k}\left|\left\langle f_{j} \mid f_{k}\right\rangle\right|^{2}\left(p_{1}-D_{j}\right)\left(p_{1}-D_{k}\right)
$$

This can be seen by noting that

$$
\begin{aligned}
\sum_{j k}\left|\left\langle f_{j} \mid f_{k}\right\rangle\right|^{2}\left(p_{1}-D_{j}\right)\left(p_{1}-D_{k}\right) & =\operatorname{Tr}\left(W^{\dagger} W\right) \\
& \geqq 0
\end{aligned}
$$


with the Hermitian operator

$$
W=\sum_{j}\left|f_{j}\right\rangle\left\langle f_{j}\right|\left(p_{1}-D_{j}\right),
$$

which concludes the proof. We note that application of the concavity of the square root above implies that also $C_{D}^{2}$ is a monotone in this case.

\section{Examples for states violating the monotone property}

As we have mentioned in Sec. 2.2, it is possible to find examples violating the monotone conditions, Eqs. (28), (29), which implies that $C_{D}$ cannot be an entanglement monotone for the local dimension in question, and all higher dimensions as well.

A numerical strategy for finding such counterexamples for $d=4$ is the following. We can restrict our attention to channels which are diagonal in the Schmidt basis of the first party. Then, the decomposition $|\psi\rangle=\sum_{j}\left|e_{j} f_{j}\right\rangle$ from Sec. 2.2 becomes the Schmidt decomposition $|\psi\rangle=\sum_{j} \sqrt{\lambda_{j}}\left|e_{j} \bar{f}_{j}\right\rangle$ with orthonormal $\left\{\left|\bar{f}_{j}\right\rangle\right\}$ and $\sum_{j} \lambda_{j}=1$. A strategy to look for parameters to violate Eq. (29) is to construct a matrix $F_{k j j k} \equiv \lambda_{j} \lambda_{k} \bar{F}_{k j j k}$ (where $\bar{F}_{k j j k} \equiv$ $\left.\left\langle\bar{f}_{k}\left|\left(\left|\widetilde{\left.\bar{f}_{j}\right\rangle\left\langle\bar{f}_{j}\right|}\right|\right)\right| \bar{f}_{k}\right\rangle\right)$ with sufficiently extremal entries, that is, with values close to 0 and others of order 1.

A matrix $\bar{F}_{k j j k}$ with such extremal entries can be found as follows. First, we generate a state $\psi_{1}$ with random entries and apply the state inverter to the projector $\Pi_{\psi_{1}}$. Since we consider an odd number of parties, the state $\psi_{1}$ is an eigenstate of $\tilde{\Pi}_{\psi_{1}}$ with eigenvalue 0 . In general, the other eigenvalues of $\tilde{\Pi}_{\psi_{1}}$ are distributed between 0 and numbers of order 1 , so that we can choose three of those other eigenstates of $\tilde{\Pi}_{\psi_{1}}$ with suffienctly different eigenvalues. Once we have computed the matrix $\bar{F}_{k j j k}$ we can perform a random search for Schmidt coefficients $\left\{\lambda_{j}\right\}$ and diagonal entries of the channel $\left\{D_{j}\right\}$ and readily find numerical examples that violate the monotone condition for $C_{D}$ or $C_{D}^{2}$. The same procedure can be applied for $d=3$ in order to find a counterexample for the monotone condition of $C_{D}^{2}$.

To conclude this part, we give an explicit analytical counterexample for a four-party state (with local dimensions $4,2,2,2$ ) that violates the monotone condition both for $C_{D}$ and $C_{D}^{2}$. It reads

$$
\begin{aligned}
\left|\psi_{4222}\right\rangle=\frac{1}{\sqrt{8}} & (|0000\rangle+|0011\rangle+|1100\rangle+ \\
& +|1111\rangle+|2000\rangle-|2011\rangle- \\
& -|3100\rangle+|3111\rangle) .
\end{aligned}
$$

The local channel acting on the first party is given by the projectors

$$
\begin{aligned}
& \mathcal{A}_{1}=\operatorname{diag}(1,1,0,0) \\
& \mathcal{A}_{2}=\operatorname{diag}(0,0,1,1) .
\end{aligned}
$$

\section{References}

[1] E. Schrödinger, Die gegenwärtige Situation in der Quantenmechanik, Naturwissenschaften 23 (49), 53 (1935).

[2] A. Peres, Quantum Theory: Concepts and Methods, (Kluwer Academic Publishers, New York, 2002).

[3] H.M. Wiseman, S.J. Jones, and A.C. Doherty, Steering, Entanglement, Nonlocality, and the Einstein-Podolsky-Rosen Paradox, Phys. Rev. Lett. 98, 140402 (2007).

[4] R.F. Werner, Quantum states with EinsteinPodolsky-Rosen correlations admitting a hidden-variable model, Phys. Rev. A 40, 4277 (1989).

[5] N. Brunner, D. Cavalcanti, S. Pironio, V. Scarani, and S. Wehner, Bell nonlocality, Rev. Mod. Phys. 86, 419 (2014).

[6] R. Horodecki, P. Horodecki, M. Horodecki, and K. Horodecki, Quantum entanglement, Rev. Mod. Phys. 81, 865 (2009).

[7] C. Eltschka and J. Siewert, Quantifying entanglement resources, J. Phys. A: Math. Theor. 47, 424005 (2014).

[8] A. Streltsov, Quantum Correlations Beyond Entanglement, SpringerBriefs in Physics, (Springer International Publishing, 2015).

[9] G. Adesso, C.R. Bromley, and M. Cianciaruso, Measures and applications of quantum correlations, J. Phys. A: Math. Theor. 49, 473001 (2016).

[10] V. Coffman, J. Kundu, and W.K. Wootters, Distributed entanglement, Phys. Rev. A 61, 052306 (2000). 
[11] M. Koashi and A. Winter, Monogamy of entanglement and other correlations, Phys. Rev. A 69, 022309 (2004).

[12] T.J. Osborne and F. Verstraete, General Monogamy Inequality for Bipartite Qubit Entanglement, Phys. Rev. Lett. 96, 220503, (2006).

[13] Y.-K. Bai, Y.-F. Xu, and Z.D. Wang, General Monogamy Relation for the Entanglement of Formation in Multiqubit Systems, Phys. Rev. Lett. 113, 100503 (2014).

[14] B. Regula, S. Di Martino, S.-J. Lee, and G. Adesso, Strong monogamy conjecture for multiqubit entanglement: The four-qubit case, Phys. Rev. Lett. 113, 110501 (2014).

[15] H.S. Dhar, A. Kumar Pal, D. Rakshit, A. Sen De, and U. Sen, Monogamy of quantum correlations - a review, Lectures on General Quantum Correlations and their Applications, Springer International Publishing, 23 (2017).

[16] B. Toner, Monogamy of nonlocal correlations, Proc. R. Soc. A 465, 59 (2009).

[17] M.P. Seevinck, Monogamy of Correlations vs. Monogamy of Entanglement Quant. Inf. Proc. 9, 273 (2010).

[18] C. Eltschka and J. Siewert, Monogamy equalities for qubit entanglement from Lorentz invariance, Phys. Rev. Lett. 114, 140402 (2015).

[19] A.A. Klyachko, Quantum marginal problem and $N$-representability, J. Phys.: Conf. Ser. 36, 72 (2006).

[20] A. Wong and N. Christensen, Potential multiparticle entanglement measure, Phys. Rev. A 63, 044301 (2001).

[21] M. Horodecki and P. Horodecki, Reduction criterion of separability and limits for a class of distillation protocols, Phys. Rev. A 59, 4206 (1999).

[22] P. Rungta, V. Buzek, C.M. Caves, M. Hillery, and G.J. Milburn, Universal state inversion and concurrence in arbitrary dimensions, Phys. Rev. A 64, 042315 (2001).

[23] W. Hall, Multipartite reduction criteria for separability, Phys. Rev. A 72, 022311 (2005).
[24] W. Hall, A new criterion for indecomposability of positive maps, J. Phys. A: Math. Gen. 39, 14119 (2006).

[25] H.-P. Breuer, Optimal Entanglement Criterion for Mixed Quantum States, Phys. Rev. Lett. 97, 080501 (2006).

[26] M. Lewenstein, R. Augusiak, D. Chruściński, S. Rana, and J. Samsonowicz, Sufficient separability criteria and linear maps, Phys. Rev. A 93, 042335 (2016).

[27] W.K. Wootters, Entanglement of Formation of an Arbitrary State of Two Qubits, Phys. Rev. Lett. 80, 2245 (1998).

[28] H. Georgi, Lie algebras in particle physics, Frontiers in physics, vol. 54. (AddisonWesley, Redwood City, 1982).

[29] S. Albeverio and S.-M. Fei, A note on invariants and entanglements, J. Opt. B 3, 223 (2001).

[30] S.J. Akhtarshenas, Concurrence vectors in arbitrary multipartite quantum systems, J. Phys. A: Math. Gen. 38, 6777 (2005).

[31] Y.-Q. Li and G.-Q. Zhu, Concurrence vectors for entanglement of high-dimensional systems, Front. Phys. China 3, 250 (2008).

[32] G. Vidal, Entanglement monotones, J. Mod. Opt. 47, 355 (2000).

[33] W. Dür, G. Vidal, and J.I. Cirac, Three qubits can be entangled in two different ways, Phys. Rev. A 62, 062314 (2000).

[34] A. Uhlmann, Roofs and convexity, Entropy 12, 1799 (2010).

[35] C. Eltschka, T. Bastin, A. Osterloh, and J. Siewert, Multipartite-entanglement monotones and polynomial invariants, Phys. Rev. A 85, 022301, (2012); Erratum, ibid., 059903 (2012).

[36] J.-M. Cai, Z.-W. Zhou, S. Zhang, and G.C. Guo, Compatibility conditions from multipartite entanglement measures, Phys. Rev. A 75, 052324 (2007).

[37] E.H. Lieb and M.B. Ruskai, Proof of the strong subadditivity of quantum-mechanical entropy, J. Math. Phys. 14, 1938 (1973).

[38] M.D. Crossley, Essential topology, Springer Undergraduate Mathematics Series (Springer, London, 2005). 